\title{
Lymphoepithelial Cyst of the Pancreas
}

\author{
Hiromitsu Domen $^{a}$ Masanori Ohara ${ }^{a}$ Noriko Kimura $^{b}$ \\ Mizuna Takahashi ${ }^{\mathrm{a}}$ Takumi Yamabuki $^{\mathrm{a}}$ Kazuteru Komuro $^{\mathrm{a}}$ \\ Nozomu Iwashiro ${ }^{a}$ Masanori Ishizaka ${ }^{a}$ \\ Departments of a Surgery and ${ }^{b}$ Pathology, National Hospital Organization \\ Hakodate Hospital, Hakodate, Japan
}

\section{Key Words}

Lymphoepithelial cyst · Pancreas · Pancreatic tumor · Endoscopic ultrasound

\begin{abstract}
Cystic lesions of the pancreas can be divided into true cysts, pseudocysts, and cystic neoplasms. Lymphoepithelial cysts (LECs) are a type of true cyst that can mimic pseudocysts and cystic neoplasms. LECs are rare lesions; fewer than 90 cases have been reported in the English language literature. The case of a 60 -year-old man with an LEC of the pancreas is reported. He was admitted with upper abdominal discomfort. Computed tomography showed a $64 \times 39 \mathrm{~mm}$ cystic mass in the retroperitoneum behind the duodenum and inferior caval vein. Magnetic resonance imaging revealed a right-sided mass on T1-weighted imaging, with a clear boundary between the mass and its surroundings, except for the pancreas. The mass had an inhomogeneous intensity on T2-weighted imaging. Within the mass, small floating nodules with low intensity were seen. Endoscopic ultrasound showed many high-echoic nodules and smaller grains scattered everywhere in the mass. Fine needle aspiration and cytologic examination were performed. Characteristic chylaceous fluid was obtained in which anucleate squamous cells were found. There were also a few atypical large cells with irregularly shaped marked nucleoli and degenerative cytoplasm. Cytologic diagnosis was suspicious for malignancy. The lesion was diagnosed as a retroperitoneal cyst, probably of pancreatic origin. Since a neoplastic lesion could not be ruled out, surgery was performed. The lesion was palpable on the dorsal side of the second portion of the duodenum. The mass was completely resected. Macroscopically, the lesion was a multilocular cyst with a thin septal wall. The cyst was filled with cottage cheese-like substance. Microscopically, the cyst wall was composed of stratified squamous epithelium and dense subepithelial lymphatic tissue with developed lymph follicles. The epithelial cells had no atypia. The histopathologic diagnosis was LEC of the pancreas. The patient's postoperative course was good.
\end{abstract}




\section{Introduction}

Cystic lesions of the pancreas are relatively rare, but they constitute an important category with a challenging differential diagnosis at the clinical, radiological and pathologic levels. Cystic lesions of the pancreas can be divided into true cysts, pseudocysts, and cystic neoplasms. Pseudocysts are the most common cystic lesions of the pancreas and usually occur as a complication of pancreatitis. True cysts, on the other hand, are most often neoplastic. A true cyst is distinguished by the presence of an epithelial lining, its benign natural history and its developmental origin. Lymphoepithelial cysts (LECs) are a type of true cyst that can mimic pseudocysts and cystic neoplasms. LECs are unilocular or multilocular cystic lesions lined predominantly by mature squamous epithelium and surrounded by non-neoplastic lymphoid elements. LECs are very rare, non-malignant lesions that were first described by Lüchtrath and Schriefers in 1985 [1]. Since then, fewer than 90 cases have been reported in the English language literature. The frequency of LECs in relation to other pancreatic cysts is largely unknown, and their clinical and pathologic features have not yet been fully characterized. We report a case of LEC of the pancreas and review the world literature.

\section{Case Report}

A 60-year-old man was admitted with upper abdominal discomfort. He had a medical history of hypertension, angina, rheumatoid arthritis and interstitial pneumonitis and took medications, i.e. an antiplatelet agent and steroid. Physical examination findings revealed no tenderness of the abdomen. Laboratory tests showed: erythrocyte count $451 \times 10^{4} / \mathrm{mm}^{3}$ (normal $410-530$ ), hemoglobin $14.1 \mathrm{~g} / \mathrm{dl}$ (normal 14-18), leukocyte count 10,300/ $\mathrm{mm}^{3}$ (normal 4,000-8,000), platelet count $19.1 \times 10^{4} / \mathrm{mm}^{3}$ (normal 15-40), serum total protein $6.6 \mathrm{~g} / \mathrm{dl}$ (normal 6.5-8.0), total bilirubin $0.62 \mathrm{mg} / \mathrm{dl}$ (normal 0.2-1.0), aspartate aminotransferase $21 \mathrm{IU} / \mathrm{l}$ (normal 10-40), alanine aminotransferase 23 IU/l (normal 5-40), alkaline phosphatase 196 IU/l (normal 115-360), $\gamma$-glutamyl transpeptidase $13.0 \mathrm{IU} / \mathrm{l}$ (normal 10-60), serum amylase $66 \mathrm{U} / \mathrm{l}$ (normal 40-132), blood urea nitrogen $15.0 \mathrm{mg} / \mathrm{dl}$ (normal 8.0-22.0), creatinine $0.79 \mathrm{mg} / \mathrm{dl}$ (normal 0.1-1.2), C-reactive protein $0.12 \mathrm{mg} / \mathrm{dl}$ (normal 0-0.3), carcinoembryonic antigen (CEA) $2.7 \mathrm{ng} / \mathrm{ml}$ (normal 0-2.5), and carbohydrate antigen (CA19-9) $43.3 \mathrm{U} / \mathrm{ml}$ (normal 0-37). Upper gastrointestinal endoscopy showed no abnormal findings. On computed tomography (CT), a cystic mass, measuring $64 \times 39 \mathrm{~mm}$, was seen in the retroperitoneum behind the duodenum and pancreas (fig. 1a). The mass had a smooth margin and was apparently well-circumscribed. The left side of the mass showed low density and the wall was slightly enhanced. On magnetic resonance imaging, an inhomogeneous signal was seen in the right side of the mass on T1-weighted imaging. There was a clear boundary between the mass and its surroundings, except for the pancreas (fig. 1b). The mass had an inhomogeneous intensity on T2-weighted imaging. Within the mass, small floating nodules of low intensity were seen (fig. 1c). Endoscopic ultrasound (EUS) imaging showed many high-echoic nodules and smaller grains scattered everywhere (fig. 1d). There was no continuity between the lesion and the gallbladder. Whether the lesions originated from the pancreas was unclear. Under EUS observation, fine needle aspiration (FNA) was performed, followed by cytologic examination. The cytologic specimen was chylaceous and contained abundant degenerated or necrotic substances. There were also scattered large cells with irregularly shaped nuclei of uncertain origin. The lesion was diagnosed as a retroperitoneal cyst, suspected to be of pancreatic origin. Since a neoplastic lesion could not be ruled out, surgery was performed. The lesion was palpable on the dorsal side of the second portion of the duodenum. The mass was dissected, and it became clear that it was part of the pancreas. The mass was completely resected. The patient's postoperative course was good, and he was discharged on postoperative day 14.

The excised specimen was a cystic mass, measuring $65 \times 60 \times 60 \mathrm{~mm}$ ( $\underline{\mathrm{fig} .} 2$, left). Hardness to touch differed depending on the location. The mass was a multilocular cyst filled with a cottage 
cheese-like substance (fig. 2, right). Microscopically, the lesion was contiguous to the pancreatic parenchyma and pancreatic origin (fig. $3 \mathrm{a}$ ). The cyst wall was composed of stratified squamous epithelium without cellular atypia and subepithelial dense lymphoid tissue (fig. 3b, c). In some parts, columnar cells or goblet cells were located on the surface of the squamous epithelium. Many macrophages phagocytosing the pink and amorphous substances mixed with the lymphatic tissue (fig. 3d). The cottage cheese-like substance was composed of an agglomerate of degenerated anucleate squamous cells. The lesion was diagnosed as LEC of the pancreas. Immunohistochemical investigation showed that the columnar epithelium, located on the surface of the squamous epithelium, was positive for CA19-9 (fig. 3e).

\section{Discussion}

The literature on LECs of the pancreas is limited to reports of single or small numbers of cases. Adsay et al. [2] reported a clinicopathologic analysis of 12 patients with LEC. In this report, the epidemiology of this disease was described as follows. LECs of the pancreas are very rare, constituting approximately $0.5 \%$ of pancreatic cysts. They are seen in middle-aged patients (mean age 55 years, range 35-82 years), predominantly but not exclusively in men $(\mathrm{M} / \mathrm{F}=4 / 1)$. The most common symptom at presentation is abdominal pain. Other complaints at presentation include nausea, vomiting, anorexia, weight loss, back pain, fatigue, fever and chills. Many cases are diagnosed during a work-up for other diseases. In the present case, the patient had upper abdominal discomfort. Upper gastrointestinal endoscopy showed no abnormal findings such as gastritis or duodenitis. We considered this symptom to be caused by LEC.

The traditional markers, such as CEA, CA19-9, carbohydrate antigen-125, cancer-related antigen 72-4, pS2 and mucin-like carcinoma-associated antigen, as well as fluid viscosity would be expected to be significantly lower in LECs than in mucinous neoplasms [3-5]; however, the limited experience with LECs does not confirm this prediction. Some cases had high CA19-9 levels in the cyst but not in the serum [6, 7]. Since the excretory ducts of the normal pancreatic tissue and some of the epithelial cells lining the cyst were immunoreactive for CA19-9, it can be concluded that CA19-9 in the cyst contents is probably produced by cells derived from the exocrine pancreas [7]. This is an interesting finding, considering that squamous metaplasia in pancreatic ductal epithelium is often immunohistochemically negative for these markers [2]. In the present case, many of the columnar epithelial cells and goblet cells located on the surface of the squamous epithelium lining the cyst were immunoreactive for CA19-9. On the other hand, the squamous epithelial cells were all negative for CA19-9.

LECs are often round and have a well-defined wall that is sharply demarcated from the pancreas and surrounding adipose tissue [2]. The lesion often protrudes from the pancreatic parenchyma, and many cases appear to be peripancreatic rather than intrapancreatic [8], as in the present case. The mean size of LECs is $4.7 \mathrm{~cm}$ (range $1.2-17 \mathrm{~cm}$ ), and they are either unilocular (40\%) or multilocular (60\%) [9]. In the present case, the lesion was a $6.5 \mathrm{~cm}$, multilocular cyst. The main radiographic finding that may help distinguish LECs is that they are sharply demarcated from the pancreatic tissue [8]. Cyst contents, which are mostly composed of keratin, have a different radiographic density than the mucin of mucinous cystic neoplasms; however, they may be difficult to distinguish from those of pseudocysts. This is true for the macroscopic appearance as well. The cyst contents display a 'cheesy' or 'caseous' appearance 
characteristic of keratinaceous debris or may be clear and serous in some cases [2]. In the present case, the lesion seemed to be a simple cyst on CT, but EUS showed a mosaic appearance inside the cyst. The cyst contents of the resected specimen seemed cheesy macroscopically.

Microscopically, LECs are characterized by cysts lined by stratified squamous epithelium and immediately adjacent, dense subepithelial lymphoid tissue that contains lymphoid follicles [2]. In some areas, the lining may appear more transitional and in others, flat, cuboidal and focally denuded [2]. The origin of pancreatic LECs is uncertain, with several possibilities suggested, including origin from epithelial inclusions in peripancreatic lymph nodes (although such inclusions have only exceptionally been demonstrated and the lymphoid elements of most LECs do not have the features of a residual lymph node), squamous metaplasia of the pancreatic ductal epithelium, derivation as a form of monodermal teratoma, and displacement of a branchial cleft cyst [9]. Another intriguing suggestion is that there is an interaction between a subset of lymphocytes and the pancreatic ductal epithelium that results in squamous differentiation and proliferation, with cyst formation initially due to secretory activity of the lining cells [2].

For many purely cystic lesions, cytologic differentiation can be difficult because of acellular aspirates [10]. However, for LECs, at least 20 cases have been reported in whom the diagnosis was made based on CT or EUS-FNA [11-15]. Cytology classically shows abundant anucleate squamous cells, multinucleated giant cells, mature lymphocytes on a background of keratinaceous debris, and a lack of neoplastic cells $[11,13,15]$. Interpretation of LECs by EUS-FNA is complicated by frequent contamination of the aspirate by tissues acquired by the needle during the procedure, such as mucinous and glandular epithelium from intestinal sources, making cystic neoplasm difficult to rule out [10]. Very often, LECs can even contain a thick milky, creamy or frothy aspirate, further confusing the diagnosis [12]. We performed EUS-FNA and obtained characteristic chylaceous fluid containing abundant anucleate squamous cells with few atypical cells. Those atypical cells are considered to be activated macrophages retrospectively. Mature or immature lymphocytes were not observed in our specimen. Chylaceous fluid with numerous anucleate squamous cells from pancreatic cyst strongly suggest LECs from our case. Chemical analysis of aspirated cyst fluid has proven to be useful in the differential diagnosis of pancreatic cysts in general [2]. However, since this disease was not suspected, a chemical analysis was not done, and a diagnosis of a pancreatic LEC could not been made preoperatively.

No recurrences or progression into lymphoma or carcinoma have been documented in the cases of LECs for whom follow-up information was available. Whatever the mechanism of formation, pancreatic LECs are benign lesions that are cured by conservative resection. Therefore, if the tumor can be diagnosed preoperatively, the option of 'wait and watch' may be clinically acceptable [2]. However, in most cases, the possibility of another type of pancreatic cystic neoplasm is difficult to rule out with the current investigative methods [2]. The difficulty in preoperative diagnosis still leads many LECs to surgical resection. Also, because LECs are often well delineated from the pancreatic parenchyma, they may seem amenable to cystectomy. However, before such an operation can be attempted, the diagnosis needs to be confirmed, because this would be a suboptimal therapy for most other macrocystic neoplasms. 


\section{Conclusion}

Although LECs are an extremely rare, physicians should bear in mind the possibility of a LEC when they detect a cystic lesion located on the surface of and associated with the pancreas. Since the prognosis of LEC is good, an appropriate strategy for its diagnosis is necessary. EUS-FNA may be helpful in the diagnosis of pancreatic cystic lesions. If malignancy cannot be ruled out preoperatively, surgery is needed. If LEC is suspected, extended surgery should be avoided; instead, minimal resection of the cyst should be performed if possible.

\section{Disclosure Statement}

The authors have no funding or conflicts of interest to disclose.
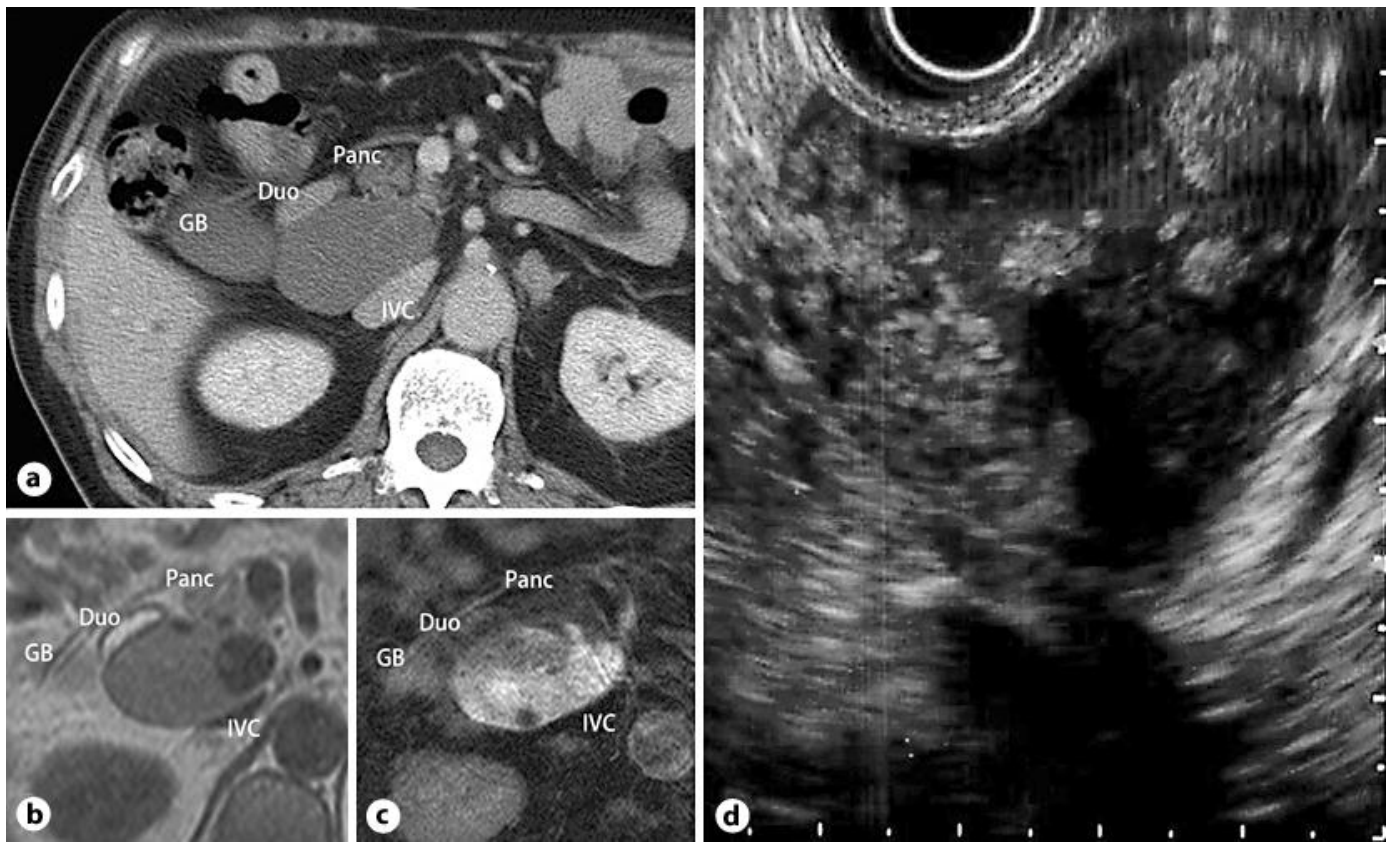

Fig. 1. CT (a), magnetic resonance imaging (b: T1-weighted image; c: T2-weighted image) and EUS imaging (d). Duo = Duodenum; Panc = pancreas; $G B=$ gallbladder; $I V C=$ inferior vena cava. a The cystic mass, $64 \times 39 \mathrm{~mm}$, is seen in the retroperitoneal area behind the duodenum and pancreas. The mass has a smooth margin and is apparently well-circumscribed. The left side of the mass shows low density and the wall is slightly enhanced. $\mathbf{b}$ On the right side of the mass, an inhomogeneous signal is seen on T1-weighted imaging. There is a clear boundary between the mass and its surroundings, except for the pancreas. $c$ The mass has an inhomogeneous intensity on T2-weighted imaging. Within the mass, small floating nodules with low intensity are seen. $\mathbf{d}$ Many high-echoic nodules are seen and smaller grains are scattered throughout. There is no continuity with the gallbladder and no evidence that the lesion involves part of the pancreas. 


\begin{tabular}{r|l|l|l}
$\begin{array}{r}\text { Case Reports in } \\
\text { Gastroenterology }\end{array}$ & $\begin{array}{l}\text { Case Rep Gastroenterol 2012;6:604-611 } \\
\text { DOI: 10.1159/000343421 }\end{array}$ & $\begin{array}{l}\text { Published online: } \\
\text { September 24, 2012 }\end{array}$ & $\begin{array}{l}\text { @ 2012 S. Karger AG, Basel } \\
\text { ISSN 1662-0631 } \\
\text { www.karger.com/crg }\end{array}$ \\
\hline
\end{tabular}

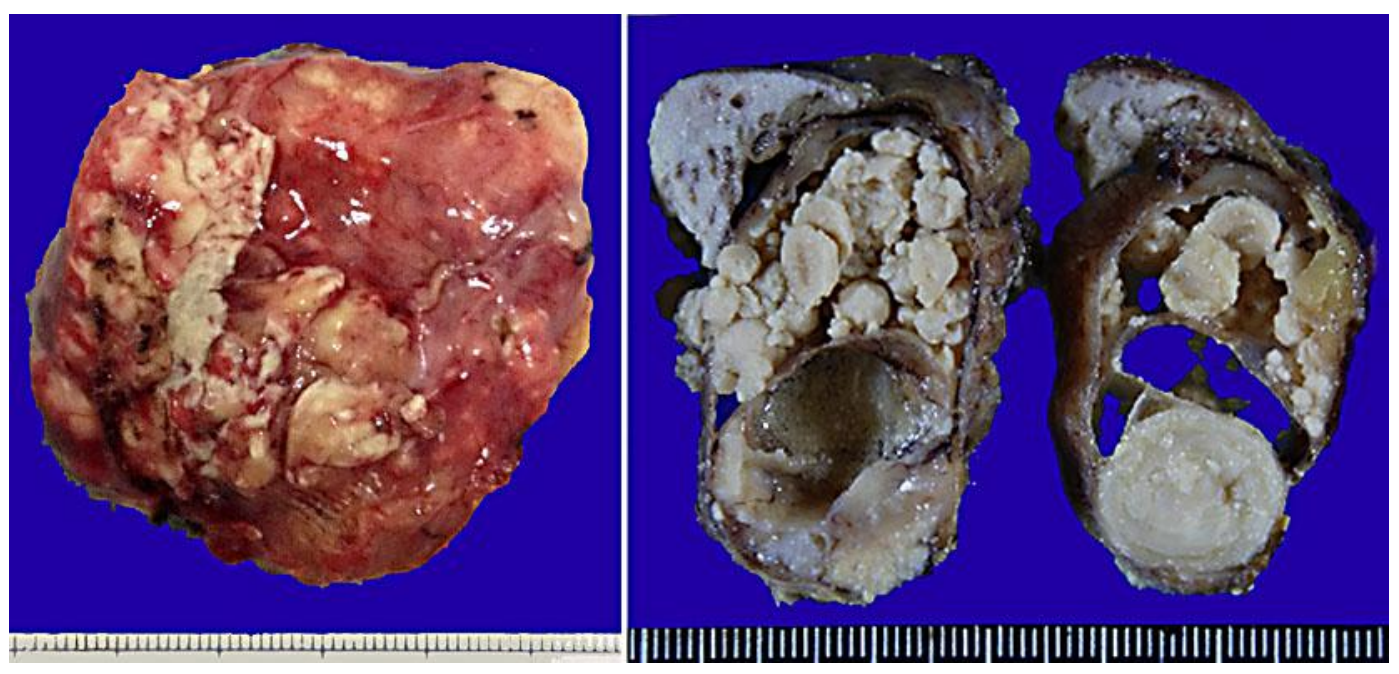

Fig. 2. Macroscopic findings. Left: Excised specimen, non-fixed. A $65 \times 60 \times 60 \mathrm{~mm}$ cystic mass is seen. Hardness to touch differed depending on the location. Right: Formalin-fixed specimen. The mass has a thin partition wall and is multilocular. The cyst is filled with a cottage cheese-like substance. 


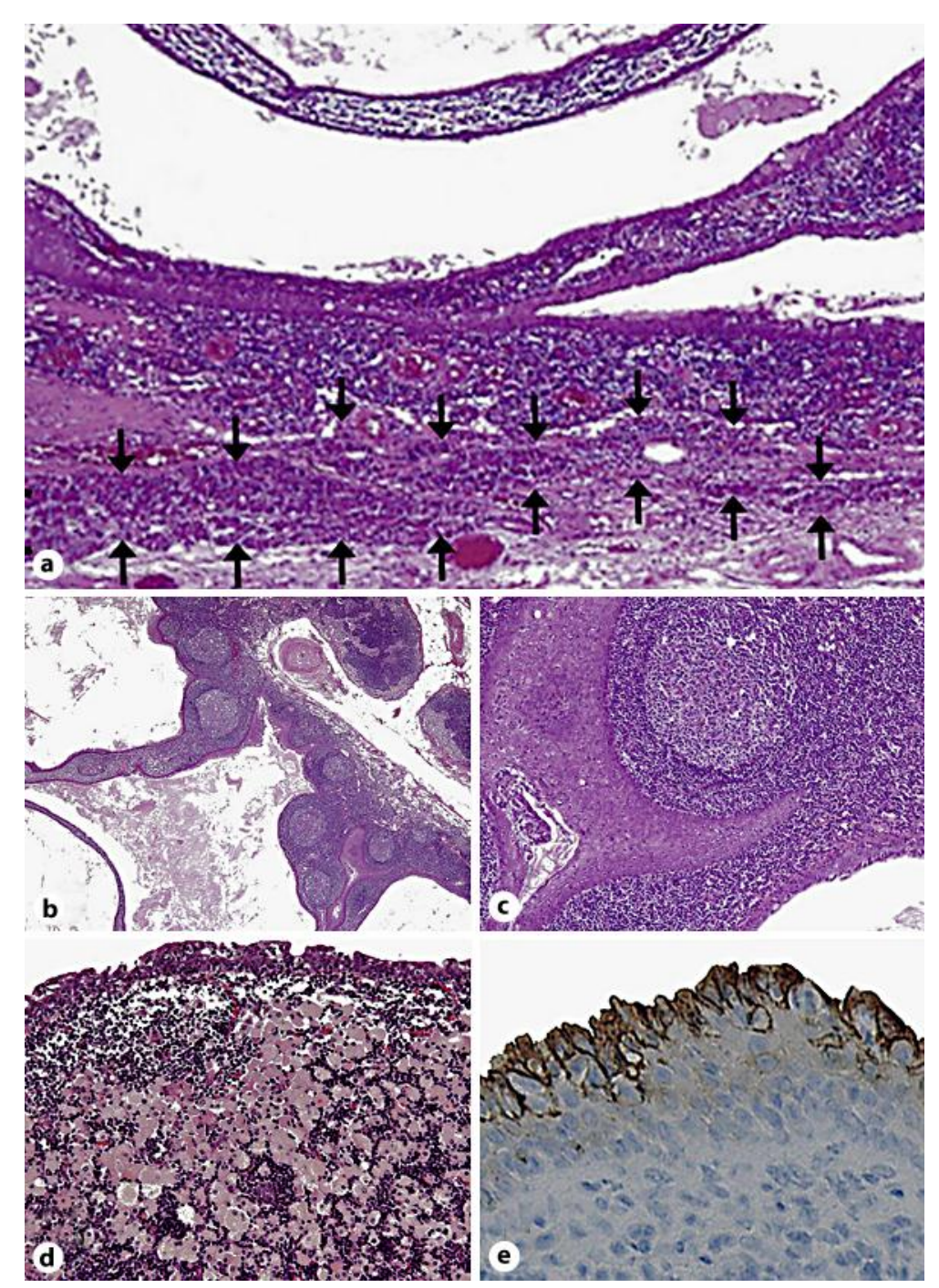

Fig. 3. Microscopic findings. a Pancreatic tissue is shown by black arrows. The lesion was contiguous to the pancreatic parenchyma and pancreatic origin $(\mathrm{HE}, \times 100)$. $\mathbf{b}$ The lesion had septal walls with abundant lymph follicles (HE, $\times 40$ ). c The cyst wall was composed of stratified squamous epithelium without cellular atypia and subepithelial dense lymphoid tissue. In some parts, columnar cells or goblet cells were located on the surface of the squamous epithelium $(\mathrm{HE}, \times 100)$. $d$ Many macrophages are seen phagocytosing the pink and amorphous granules mixed with lymphatic tissue (HE, $\times 200)$. e The columnar epithelium located on the surface of squamous epithelium was immunohistochemically positive for CA19-9 $(\times 400)$. 


\section{References}

1 Lüchtrath H, Schriefers KH: A pancreatic cyst with features of a so-called branchiogenic cyst (in German). Pathologe 1985;6:217-219.

2 Adsay NV, Hasteh F, Cheng JD, Bejarano PA, Lauwers GY, Batts KP, Kloppel G, Klimstra DS: Lymphoepithelial cysts of the pancreas: a report of 12 cases and a review of the literature. Mod Pathol 2002;15:492-501.

3 Adsay NV, Hasteh F, Cheng JD, Klimstra DS: Squamous-lined cysts of the pancreas: lymphoepithelial cysts, dermoid cysts (teratomas), and accessory-splenic epidermoid cysts. Semin Diagn Pathol 2000;17:56-65.

4 Lewandrowski KB, Southern JF, Pins MR, Compton CC, Warshaw AL: Cyst fluid analysis in the differential diagnosis of pancreatic cysts. A comparison of pseudocysts, serous cystadenomas, mucinous cystic neoplasms, and mucinous cystadenocarcinoma. Ann Surg 1993;217:41-47.

5 Yang JM, Southern JF, Warshaw AL, Lewandrowski KB: Proliferation tissue polypeptide antigen distinguishes malignant mucinous cystadenocarcinomas from benign cystic tumors and pseudocysts. Am J Surg 1996;171:126-129; discussion 129-130.

6 Chan SP, Hatton CW, Falk GL: Lymphoepithelial cyst of the pancreas: serum markers do not help. HPB Surg 1998;11:121-124.

7 Kaiserling E, Seitz KH, Rettenmaier G, Seidel W, Kahlfuss R, Walz-Mattmuller R, Becker V: Lymphoepithelial cyst of the pancreas. Clinical, morphological, and immunohistochemical findings. Zentralbl Pathol 1991;137:431-438.

8 Kazumori H, Sizuku T, Ueki T, Uchida Y, Yamamoto S: Lymphoepithelial cyst of the pancreas. J Gastroenterol 1997;32:700-703.

9 Hruban RH, Pitman MB, Klimstra DS; American Registry of Pathology; Armed Forces Institute of Pathology: Tumors of the pancreas; in: AFIP Atlas of Tumor Pathology, fourth series, fasc 6. Washington, DC, American Registry of Pathology in collaboration with the Armed Forces Institute of Pathology, 2007.

10 Karim Z, Walker B, Lam E: Lymphoepithelial cysts of the pancreas: the use of endoscopic ultrasoundguided fine-needle aspiration in diagnosis. Can J Gastroenterol 2010;24:348-350.

11 Mandavilli SR, Port J, Ali SZ: Lymphoepithelial cyst (LEC) of the pancreas: cytomorphology and differential diagnosis on fine-needle aspiration (FNA). Diagn Cytopathol 1999;20:371-374.

12 Nasr J, Sanders M, Fasanella K, Khalid A, McGrath K: Lymphoepithelial cysts of the pancreas: an EUS case series. Gastrointest Endosc 2008;68:170-173.

13 Ahlawat SK: Lymphoepithelial cyst of pancreas. Role of endoscopic ultrasound guided fine needle aspiration. JOP 2008;9:230-234.

14 Renou C, Giovannini M, Monges G, Rifflet H, Muller P, Harafa A, Martini F, Benderitter T, Igual JP, Delpero JR, Laugier R: Pitfalls of cyst fluid findings obtained by endoscopic ultrasonography fine-needle aspiration on a pancreatic lymphoepithelial cyst. Am J Gastroenterol 2007;102:213-215.

15 Zou XP, Li YM, Li ZS, Xu GM: Lymphoepithelial cyst of the pancreas: a case report. Hepatobiliary Pancreat Dis Int 2004;3:155-157. 